\title{
1. Introduction to the Field Guide for Research in Community Settings: Tools, Methods, Challenges and Strategies
}

\author{
M. Rezaul Islam, Niaz Ahmed Khan, Siti \\ Hajar Abu Bakar Ah, Haris Abd Wahab and \\ Mashitah Binti Hamidi
}

Fieldwork/data collection is one of the most important parts in the research process, and it is particularly important for social sciences research. A number of aspects that need to be considered by a researcher before starting data collection include: ethical permission from the concerned ethical body/committee, informed consent, contract with different stakeholders, field settings, time allocation and time management, field leading, data collection, contextual and cultural diversities, community settings, socioeconomic and psychological patterns of the community, political pattern, rapport building between data collectors and respondents, permission to access community, language and mode of data collection, power relations, role of gatekeepers, privacy and confidentiality issues, layers of expectations among researchers/respondents/ funding organization, data recording (written, memorization, voice recording and video recording), and so on. Many aspects are very difficult to understand before going into the field. Sometimes, a researcher's previous experience about a particular community may help to gain field access, but it may be difficult to assess the field in advance due to rapid changes within people's livelihoods and other shifts in the community. The change of a political paradigm sometimes seems also to be a challenge at the field level. We believe that although technological innovation has benefited some aspects of the data collection of fieldwork in social research, many other dimensions (mentioned above) of fieldwork endure unchanged. It is true that the improvement of technology is no promise that the quality of fieldwork will improve. However, the orbit of fieldwork has always had to deal with the shifting nature of societies themselves (Porter and Grossman, 2004). 
There are a number of issues that can be challenges at the field level for the data collection process, though this edited volume strongly argues that the local context is the prime and leading consideration for such challenges that researchers should take seriously. One important issue within this spectrum is ethical consideration relating particularly to 'harms and benefits' (Cassell, 1982). In many cases, particularly data collection in low-income communities through participatory research, the role of social advocacy and empowerment of the poor and the powerless is very important (Porter and Grossman, 2004). On the other hand, in developing countries, there has been an expansion in forbidden or restricted research due to the stimulation of privatization and human subject regulations (Warren and Staples, 1989). In qualitative study, such as anthropology, there has been much debate as to whether such research (mainly through observation) is a real science. This became a critical issue when Malinowski and Radcliffe-Brown strongly argued that anthropological research is also a science like experimental research (Jarvie, 1986). This volume is a breakthrough that provides many examples from diverse fields in terms of the nature and types of communities, cross-country and ethnic diversity, various community settings, cultural and religious beliefs, multiple socioeconomic and psychological patterns and so on. Contributions to this edited book - reflecting these diversities - prove that the basis for all new knowledge in the social sciences is the affective. This book will be an interesting read for the researcher in social sciences faced with adjusting to uncertainties. The new material in this book adds to our basic understanding of real-world difficulties.

The present volume provides many examples from the field of anthropology, demonstrating that affect is the centre of all of our knowledge-creation efforts. In this regard, George Devereux (1967) pointed to various ways in which researchers deal with uncertainties. The new material in this book adds to our basic understanding of the real-world difficulties experienced by diverse communities and of the ways in which human beings face many changes to their livelihoods. This volume provides many examples of the challenges researchers face during data collection, such as various misperceptions of their roles as outsider-researchers and qualms about their impacts on the lives of the communities they study. Many chapters show that development of trust is an essential component in fieldwork that can be considered as a fragile product but essential for effective interpersonal interaction (Stodulka et al., 2019).

We feel confident that this book will add considerable value to literature on research methodologies in social sciences by focusing on some practical dimensions of field research for advanced degrees in a way which has seldom been tried in earlier studies. The book extensively addresses a fundamental component of social science research methodology - namely fieldwork, more specifically the application of techniques and strategies of fieldwork in data collection including the issue of research ethics. One important feature of this 
book is that it explores a wide range of contextual and community settings across several countries within which the respective researchers have devised and applied context- and community-specific research approaches, data collection methods and field research instruments to suit and pursue their research objectives. In documenting the diverse experiences of the researchers in the varied contexts, this book also captures the practical challenges including ethical dilemmas faced by the researchers during the data collection process. Secondly, the authors also provide a large number of practical illustrations and examples of the strategies they adopted during fieldwork in order to overcome these challenges. Thus this book may serve as handy resource material for research students across a wide range of social and behavioural science disciplines by giving them an opportunity to learn these hands-on tips, strategies and experiences shared by fellow researchers who have already paved the way and emerged successfully! Given these strengths, the editors are optimistic that the publication may attract wide international readership.

This volume, in the main, attempts to offer some practical tips and messages in relation to fieldwork in social and behavioural sciences; the messages address such phases of empirical research as the application of research tools and techniques in data collection, the challenges commonly faced in the field, and the strategies that researchers adopt to overcome these challenges, either by using their indigenous or innovative skills or exploiting and making judicious use of the advantages already available within the standard research methodologies. The specific objectives are: (i) to examine the major challenges the researchers face during their fieldwork (data collection), (ii) to capture diverse learning across different countries and communities (local perspective and contextual aspects), and (iii) to learn about the strategies (either indigenous or within the methodological scope) the researchers followed to overcome the challenges. In general, this book will help to enrich researchers' capacity and skills, especially in the context of social science fieldwork and empirical examinations. It is often argued that social research is more effectively understood and learnt as a practice rather than through classroom briefings. This book includes 18 chapters which are written in the light of the various authors' practical field experiences.

Within its broad focus of fieldwork tools, methods, challenges and strategies in social and behavioural sciences, the book covers a very wide range of important research topics and agendas. Examples of such topics include: definitional debates of research, types and classification, philosophical aspects of research, research design and planning, research methods (for example, survey, experimental, case study, ethnographic, content analysis), data collection methods (face-to-face interview, in-depth case interviews, focus group discussions, key informants' interviews, community mapping, observation, documentation review, etc.), and data collection instruments (interview sched- 
ule, guideline, and checklist). Other issues include preparation and protocol for fieldwork, ethical issues, sampling procedures (for qualitative, quantitative and mixed-method approaches), data analysis techniques (use of SPSS, NVIVO, thematic, triangulation, etc.), research management, data management, data coding, and the qualities of social researchers. It also covers the qualities that are helpful in conducting a research project such as mental, psychological, and social factors and the need for financial as well as time management. Various ethical issues in conducting social research receive considerable attention in several chapters. The discussion also addresses research ethics from two distinct perspectives, i.e. Western and Asian, and focuses on the strategies and techniques deployed by the researchers to tackle various ethical challenges.

Secondly, the book provides many perspectives and contextual aspects related to the procedural and communal level challenges commonly faced by researchers. This is a good opportunity for the reader to examine these challenges through a wide range of examples from different communities, countries, and ethnicities. Many chapters address the issues and dilemmas of research ethics head on. The cases and examples will be of value to research students in preparing them for fieldwork and empirical data collection. Thirdly, this book has captured some practical experiences that may help research students to learn how to put into practice the method or approach that they are theoretically conversant with. It thus contributes to bridging the gaps between relevant theories and practices of social research. For example, a research student knows about the procedure, use, advantages and disadvantages of the interview method, but he or she may not yet have the practical skills to use this data collection tool in the field. A student may already have decided on the ethical guidelines that he or she will follow during data collection, but lack practical experience in how these guidelines should be followed at the field level. One more example is that a student may have knowledge about the use of ethnographic method, but not have any experience as to how it can be used among vulnerable communities in Bangladesh. This book will advise the research student on the preparation they need and pointers on how to gain practical experience as he or she uses different research tools and techniques in the field. The many examples of how to work in diverse countries and communities will surely enrich the student's research knowledge.

The book contains extensive examples of application of selected research tools in different cultural and socioeconomic contexts and communities. In a number of chapters, the authors report and analyse community experiences from Muslim and non-Muslim countries, while other chapters address the challenges arising from research among ethnic (tribal) communities. Each chapter has its own scope and limitation; the book does not follow a particular uniform template and allows the authors a degree of freedom to present their cases and experiences in a manner and format that best serve and suit the purposes of 
their respective chapters. Research students may learn the techniques and art of applying and adjusting a particular research tool in various contextual settings. Thus one can get diverse meanings and applications of a particular research method or tool adapted to changing contexts.

In many cases, the chapters of this book use verbatim quotations and dialogues from the field in order to give the contextual meaning and portray the real voices and vision of the research participants/respondents. Such an approach will surely help the readers, especially research students, to learn practical techniques of capturing field voices from their own research in an academically acceptable manner. This is particularly important for students who plan to use the qualitative research approach in their studies. From our experience of serving as advanced research degree examiners or reviewers, we noted that students often struggle with effective ways of depicting and capturing field observations and people's views, especially in qualitative research. This book can be a very useful guide for research students faced with these challenges.

Another important approach and feature of this book is that it follows a 'storytelling' method in delivering and understanding the meanings and perspectives of the research subjects. Research students may be particularly interested to hear the stories from different countries at various levels - community, group and individual. We believe that this is a relatively new approach to community research. Most of the chapters expand the respective stories with analysis of particular contextual settings in order to make the presentations exciting to the readers. Most textbooks or resource materials follow a traditional form of writing that typically involves the following sequence of presentation: the dictionary meaning of a concept first, then the general meaning, followed by some refereed definitions and connotations, and finally the author(s)' view on the definition. The approach used in this book - manifested in the 'storytelling' mode - will make a departure from this conventional structure and format. Here the readers will get the concepts, their application and associated examples in a systematic manner that will help them to understand and grasp the messages and meanings more easily.

This edited volume brings together 18 chapters contributed by 41 authors from around the world. Chapter 2, 'Challenges and solutions for collecting data in health research: experiences of Australian doctoral and early career researchers' by Mohammad Hamiduzzaman and colleagues begins our journey by exploring the methodological challenges in health research in Australia. They mention that the National Health and Medical Research Council stipulates a governance process that covers standards of ethical practice and the legal responsibilities of researchers in respecting privacy and maintaining confidentiality and dignity of participants. In this connection, they argue that data collection in clinical and community settings remains challenging for 
doctoral and early career researchers due to the complexities of research governance systems and issues with the involvement of participants in research activities. The major challenges are related to research governance, expertise of researchers, health literacy of participants, fieldwork settings, approaching and designing questions, and research fatigue. In Chapter 3, 'Challenges with opening up closed off communities: interviewing ultra-Orthodox Jewish communities in Israel', Veronika Poniscjakova explores her doctoral research (at the University of Nottingham) fieldwork challenges among counter-culture communities in Israel. She examined the religious Zionist and ultra-Orthodox Jewish communities' attitudes towards political developments and crises of political and religious nature. Her challenges were primarily related to access, trust building, and language issues. She had to identify gatekeepers to introduce her to individuals who were more open-minded. This affected representation and validity but led to a snowball effect. The chapter advises readers to identify gatekeepers, communicate with interviewees in a culturally sensitive way whilst still getting good data, and understand the participants' world.

Chapter 4, 'Ethnography research with Indonesian female factory workers: challenges and strategies in the field' by Mashitah Binti Hamidi, explores the fieldwork challenges she faced with the Indonesian female migrant workers in Malaysia's manufacturing sector. She describes that this research challenged her in her role as an 'insider' doing research in her own backyard while seeking to understand at the same time as an 'outsider'. In Chapter 5, entitled "'How can you be so naïve?" Negotiating insider status among co-ethnic migrants in global ethnographic fieldwork', Hasan Mahmud addresses the data collection challenges by using the Weberian and Symbolic Interactionist approaches, two foundations of ethnographic research, which both imply that the researchers are outsiders to the groups they study and need to access the insiders' perspective. The author describes his fieldwork challenges among Bangladeshi migrants in Tokyo and Los Angeles. He begins with Merton's discussion on the insider/outsider debate from the sociology of knowledge perspective and demonstrates its epistemological importance for fieldwork. He looks at the ethnographic conceptualization of the field in the context of globalization and how migration researchers negotiate their insider/outsider role in conducting fieldwork.

A comparative study brings more data collection challenges when working with two different communities in two countries. Rajendra Baikady in Chapter 6, 'Challenges and opportunities in conducting cross-country PhD study: experiences of data collection in India and China', faced these comparative challenges while he collected data from India and China. He examined the status of social work education in a parliamentary democracy and in a communist regime. He looked at the curriculum, teaching, learning and practices, level of indigenization of social work education and practice and civil society engage- 
ment in social work education. His study examined the ontological and the epistemological assumptions that social work is a socially constructed institutional care practice where the emergence and the development of social work are largely governed by the social, economic, political and cultural context of the country. These contexts either involve or influence the development and relevance of social work. In Chapter 7, 'Researching the garment sector in Bangladesh: fieldwork challenges and responses', Sawlat Zaman discusses the data collection challenges arising from the ready-made garment (RMG) sector of Bangladesh, where much debate and controversies exist around this industry, especially due to catastrophic disasters like Spectrum Collapse, Rana Plaza, and Tazrin Fire that call into question the sector's record on fair labour practices. She discusses access, cultural and contextual challenges experienced by her doctoral research. Data collection is particularly challenging for any researcher working in a community which is comparatively remote and with a low standard of living.

This book incorporates diverse challenges from two hard-to-reach communities in Bangladesh and Nigeria. Shofiqur Rahman Chowdhury with his supervisors M. Rezaul Islam and Haris Abd Wahab capture some of these challenges in Chapter 8, 'Gaining access to research participants for data collection in doctoral studies: evidence from a rural area of Bangladesh'. The authors explore the challenges where a faith-based Islamic NGO named Islamic Relief Worldwide provided interest-free loans and training to the rural poor community under the community empowerment domain. The challenges encountered are community threat, understanding of local culture, clarifying study objectives, and conducting uninterrupted surveys and interviews. They describe how the use of different social and institutional gatekeepers, community mapping, applying local contextual examples to clarify study objectives, and recalling positive memories were effective ways to deal with the nuances in the field and data collection. Chapter 9, 'The challenges and strategies of accessing hard-to-reach locations during fieldwork data collection: the case of northeast Nigeria' by Nasa'i Muhammad Gwadabe and Adekunle Daoud Balogun, narrates and analyses the challenges such as location and accessibility, insecurity, culture and religion, duration of data collection, researchers' fatigue and sensitive information during data collection on Internally Displaced Persons (IDPs) in northeast Nigeria.

Ashek Mahmud with his supervisors M. Rezaul Islam and Hamedi M. Adnan in Chapter 10, 'Data collection on "smartphone addiction and social capital effects" among the university students of Bangladesh: challenges and strategies for the way out' relate how they faced various ethical drawbacks, sociocultural malpractices, and structural limitations to collect data from smartphone-addicted university students in Bangladesh. In Chapter 11, 'Undercover fieldwork: a queer experience of healthcare in Bangladesh', 
Kanamik Kani Khan describes his experience of $\mathrm{PhD}$ fieldwork with sexual and gender diverse communities who live invisibly in Bangladesh. He conducted in-depth case interviews, focus group discussions, and key informants' interviews where developing trust and gaining access to this invisible community were challenging and describes how building networks and recruiting participants were key strategies to overcome the challenges. Like Chapter 6 , Chapter 12 also deals with an example of cross-country fieldwork. In their chapter entitled 'Ethical issues, challenges and solutions during fieldwork with homeless elderly people of Malaysia and Pakistan', Aqsa Qandeel and her supervisor Welyne J. Jehom discuss a number of challenges arising from fieldwork with two diverse elderly groups, including their living situations, mental conditions, and tracing of respondents, interactional hesitation with a stranger, linguistic differentiation, racial difference, unfavourable response to the interviewee, being a foreigner, mood swings, and issues of interviewee femininity. In Chapter 13, 'Field research in the conflict zone: an empirical study of the Chittagong Hill Tracts (CHT) in Bangladesh', Md. Rafiqul Islam explores the data collection challenges experienced among the ethnic community in Bangladesh. This is one of the conflictual and violent zones in Bangladesh where safety, security, silence, mistrust between communities, a culture of violence, unwillingness to share information and interference of the security forces in the context of conflict research were specific data collection challenges.

In Chapter 14, 'Research with coastal people in Bangladesh: challenges and way forward', Taj Sultana and her supervisors Firuza Begham Binti Mustafa, Jillian Ooi Lean Sim and M. Rezaul Islam describe some contextual challenges of data collection using a mixed-method approach that included complexity of site accessibility impeded by tidal floods; social issues such as personal security, lack of trust between researcher and respondents, and the problem of matching official addresses to actual physical locations; and political issues that came about because data collection coincided with the national elections of 2018, creating a volatile and politically charged situation within the study area. Munira Jahan Sumi with her supervisors M. Rezaul Islam and Ramy Bulan explore the data collection challenges arising from the Santal (an ethnic) community about their land rights in Bangladesh in Chapter 15, 'Data collection from the Santal community: a journey towards an unknown world in ascertaining the nexus between reality and dream'. Most of the challenges were rooted in the Santal's traditional livelihood practices and in understanding their local context. In Chapter 16, 'Challenges in accessing rural area and managing sub-culture differences in Kuala Krai, Kelantan, Malaysia', Maria Binti Mohd Ismail and Raja Noriza Binti Raja Ariffin explore some diverse challenges such as remoteness, local accent of the language, cultural difference, and values conflict, in a remote area in Kuala Krai, Kelantan, Malaysia. 
Bushra Zaman with her supervisors M. Rezaul Islam and Rosila Bee Mohd Hussain in Chapter 17, 'Fieldwork experience: challenges and managing risks as a female researcher', explore their data collection experiences among the Bangladeshi and Indonesian labour migrants working in Malaysia. Bushra Zaman's PhD study, 'Usage of social capital among migrant workers for their livelihoods in Malaysia', examined whether social capital can bring any change in the migrant workers' livelihoods between two high labour sending countries, Indonesia and Bangladesh. She faced gender stereotyping, language barriers, safety and security issues, natural caution of migrant workers, and rejection from the key informants. In the end, leveraging the power of networking, building relationships, developing acceptance, pursuing alternative avenues to address challenges, persevering and being prepared, and following the passion and purpose of a researcher helped to minimize and manage those risks. An interesting account of data collection difficulties is presented in Chapter 18, 'Data collection on acid attack survivor women: a PhD researcher's experience from Bangladesh', by Tahmina Islam and her supervisors M. Rezaul Islam and Siti Hajar Abu Bakar Ah. The main challenges related to social and physical aspects such as the behaviour of people surrounding the acid attack survivors and the bad transport and communications system of the country. Civil society engagement and the researcher's expression of forbearance and empathy solved these problems. Finally, Chapter 19, 'Challenges, strategies, and way out techniques in conducting in-depth interviews among managers in Malaysian organizations', describes some challenges at organizational level when a $\mathrm{PhD}$ researcher (low power) attempts to get access and approval from the higher authorities (high power) to conduct her research. This power relation is discussed by Nafisa Kasem and her supervisors Shahreen Mat Nayan, Kumaran A/1 Suberamanian and Sedigheh Moghavvemi.

In conclusion, we reiterate that this book includes chapters from diverse countries, cultures, and Muslim and non-Muslim community contexts, which capture and highlight $\mathrm{PhD}$ field research experiences. This reflects the global focus of this book with potential global outreach. Research methodology is an essential and compulsory subject in nearly all faculties such as arts, social sciences, business, and medical sciences commonly taught in tertiary educational institutions. Thus the potential readership of this book may be quite diverse and wide. We believe this will be a particularly valuable resource for research students since fieldwork and data collection are integral and fundamental components of any research project.

\section{REFERENCES}

Cassell, J. (1982). Harms, benefits, wrongs, and rights in fieldwork. In J. E. Sieber (ed.), The Ethics of Social Research (pp. 7-31). New York: Springer. 
Devereux, G. (1967). From Anxiety to Method in the Behavioral Sciences, Etc. Paris: Hague.

Jarvie, I. C. (1986). On theories of fieldwork and the scientific character of social anthropology. In I. C. Jarvie, Thinking about Society: Theory and Practice (pp. 107-126). Dordrecht: Springer.

Porter, P. W. and Grossman, L. S. (2004). Fieldwork in nonwestern contexts: Continuity and change. In S. D. Brunn, S. L. Cutter, and J. W. Harrington Jr. (eds.), Geography and Technology (pp. 201-220). Dordrecht: Springer.

Stodulka, T., Dinkelaker, S., and Thajib, F. (eds.) (2019). Affective Dimensions of Fieldwork and Ethnography. Cham: Springer Nature.

Warren, C. A. and Staples, W. G. (1989). Fieldwork in forbidden terrain: The state, privatization and human subjects regulations. The American Sociologist, 20(3), 263-277. 